\title{
New Therapies for Ovarian Cancer
}

\author{
Presented by Deborah K. Armstrong, MD
}

\section{Abstract}

In the latest NCCN Guidelines for Ovarian Cancer, the histologic subtypes of ovarian cancer are described in more depth as they vary by frequency, typical age and disease stage at presentation, treatment recommendations, and survival probabilities. The less common subtypes are also discussed. The update with the greatest impact on the treatment of ovarian cancer, however, is probably the use of maintenance therapy with poly(adenosine diphosphate-ribose) polymerase (PARP) inhibitors, and 3 PARP inhibitors are now included in the guidelines. These drugs have made a large difference in outcome, both for patients with BRCA mutations and in unselected patients.

J Natl Compr Canc Netw 2018;16(5.5):632-635 doi: 10.6004/jncen.2018.0034

The NCCN Guidelines for Ovarian Cancer include recommendations for a broad range of histologic subtypes of this malignancy, and recommendations for use of oral poly(adenosine diphosphate-ribose) polymerase (PARP) inhibitors. As maintenance therapy in select patients with recurrent, platinum-sensitive, chemotherapy-responsive disease, PARP inhibitors were shown to improve progression-free survival (PFS) for patients with BRCA-mutant and BRCA wild-type disease, indicating that these agents are potentially beneficial to many patients with platinum-sensitive disease in this setting, according to Deborah K. Armstrong, MD, Professor of Oncology, The Sidney Kimmel Comprehensive Cancer Center at Johns Hopkins; Director of the Johns Hopkins Breast and Ovarian Cancer Screening Service; and Chair of the NCCN Guidelines Panel for Ovarian Cancer.

"The first time we included information about histology was in 2017, so this topic is still new. PARP inhibitors represent an explosion in the world of ovarian cancer, and they have been included in the guidelines since 2016. Now, we list 3 in the [NCCN] Compendia, and we are also emphasizing their use as maintenance

Presented by Deborah K. Armstrong, MD, The Sidney Kimmel Comprehensive Cancer Center at Johns Hopkins, Baltimore, Maryland. Dr. Armstrong has disclosed that she has served as a scientific advisor for Morphotek Inc., and has received grant/research support from Advaxis, AstraZeneca Pharmaceuticals LP, Clovis Oncology, Pfizer Inc., Syndax, and Tesaro, Inc., and that her spouse or significant other has received grant/research support from Eisai Inc. and Exelixis Inc.

Correspondence: Deborah K. Armstrong, MD, The Skip Viragh

Outpatient Cancer Building, The Sidney Kimmel Comprehensive Cancer

Center at Johns Hopkins, 201 North Broadway, 10th floor, Room 10293,

Baltimore, MD 21287.. Email: armstde@jhmi.edu therapy," Dr. Armstrong said as she described this malignancy at the NCCN 23rd Annual Conference.

\section{Expanded Description of Histologic Subtypes}

Three cell types within the ovaries can become malignant. They have different frequencies, and age range and stage at diagnosis:

- Epithelial: $80 \%-90 \%$ (of ovarian malignancies); age range, 30-79+ years; 70\% stage III/IV

- Germ cell: 5\%-10\%; age range, $<35$ years; $70 \%$ stage $\mathrm{I} / \mathrm{II}$

- Sex cord/stromal: 5\%-10\%; age range, 50-79+ years; $>80 \%$ stage $\mathrm{I} / \mathrm{II}$

"There has been a paradigm shift in that we now think most epithelial ovarian cancers originate in the fallopian tubes," Dr. Armstrong added. In the guidelines, "ovarian cancer" includes fallopian tube and primary peritoneal cancers as well.

The survival rates are different for the different subtypes, largely reflecting the stage at which they are likely to present. Both germ cell tumors and stromal cell tumors often present early and are associated with better survival than epithelial cell malignancies, which usually present at a more advanced stage.

The $8^{\text {th }}$ revision of the AJCC Staging System offers the following simplified staging of ovarian cancer, with survival rates as shown: 
- Stage I: disease limited to the ovary (15\% of patients with ovarian cancer); 5-year survival, $90 \%$

- Stage II: spread limited to the pelvis $(<5 \%)$; 5-year survival, $70 \%$

- Stage III: spread to upper abdomen (70\%); 5-year survival, 25\%-50\%

- Stage IV: distant metastases, mainly liver, pleura, liver parenchyma (10\%); 5-year survival, 10\%$15 \%$

"Fully $85 \%$ of patients have extra-ovarian disease at diagnosis. We are not good at diagnosing ovarian cancer when it is curable by surgery and limited to the ovary. Survival rates decline with more advanced disease, not unsurprisingly," Dr. Armstrong explained.

Ovarian cancer tends to fall into 2 main categories, type I (25\%) and type II (75\%), which are different pathologically and clinically and have different precursors and molecular profiles (Figure 1).

Type II tumors tend to be advanced at diagnosis and are usually aggressive. These are the tumors most commonly seen by oncologists and where most of the treatments are delivered. One quarter of patients will have type I disease, and these will be predominantly early-stage and low-grade tumors. Dr. Armstrong noted that a transition from type I to type II does not seem to occur (ie, a borderline tumor does not become a high-grade serous carcinoma).

\section{Treatment Considerations}

After aggressive surgical debulking of newly diagnosed ovarian cancer (where possible), most patients receive chemotherapy with paclitaxel and carboplatin. In early-stage nonserous epithelial ovarian cancer, 3 cycles have been shown to be as effective as 6

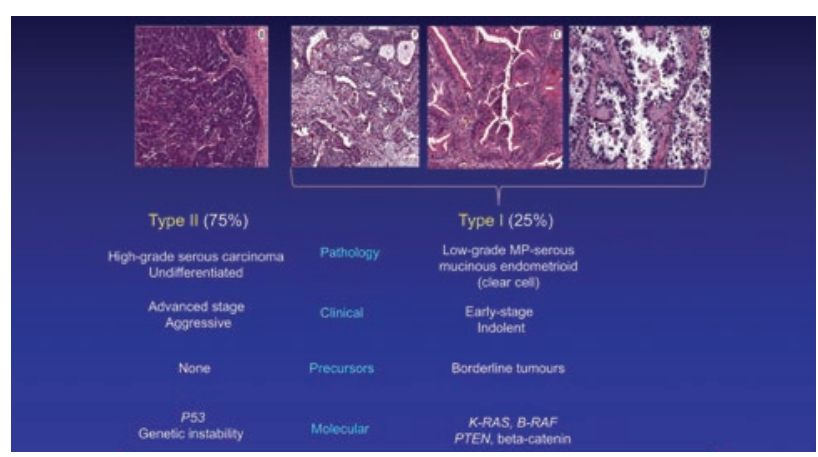

Figure 1. Characteristics of ovarian cancer subtypes. Abbreviation: MP, micropapillary. in preventing relapse; however, for serous carcinoma, 6 cycles provides better protection from relapse. This finding is mentioned in the guidelines. The NCCN Guidelines Panel for Ovarian Cancer discussed histology as it relates to duration of treatment. Serous carcinoma is most common in advanced disease; endometrioid is seen more in early-stage than advanced disease; and clear cell is almost always an early-stage subtype.

"Clear cell cancer does not respond well to chemotherapy, but it does not appear to spread as commonly as does high-grade serous, and that is something to keep in mind," Dr. Armstrong said. The guidelines for newly diagnosed patients do not, however, exempt clear cell from the standard recommendation of 3 to 6 cycles for stage I disease and 6 cycles for stage II-IV disease.

Clear cell carcinoma is among the less common histologic types for which the guidelines provide specific treatment recommendations. The panel has also provided treatment recommendations for carcinosarcoma, mucinous tumors, low-grade serous/ grade I endometrioid carcinoma, malignant germ cell tumors, stage Ib-III resected dysgerminoma, and malignant sex cord/stromal tumors.

\section{BRCA Mutations and PARP Inhibitors}

BRCA mutations occur in approximately $15 \%$ of patients with ovarian cancer, and this subgroup has been shown to be more likely to respond to PARP inhibitors. The molecular profile of ovarian cancer is therefore important, and all women with ovarian cancer should undergo genetic counseling with "strong consideration" for genetic testing, she said.

Due to their efficacy in ovarian cancer and some other malignancies, the use of PARP inhibitors has steadily grown since 2014, when olaparib was approved. At the time of Dr. Armstrong's talk, the FDA-approved indications included the following for ovarian cancer:

- Olaparib (treatment of active disease) was approved December 19, 2014, for patients with deleterious or suspected deleterious germline BRCA-mutated advanced ovarian cancer after $\geq 3$ prior lines of therapy.

- Rucaparib (treatment of active disease) was approved December 19, 2016, for patients with 
deleterious germline or somatic BRCA-mutation after $\geq 2$ prior lines of therapy. [Editor's note: rucaparib was approved as maintenance therapy after the NCCN Conference.]

- Niraparib (maintenance therapy) was approved March 27, 2017, as maintenance for recurrent ovarian cancer for patients who are in complete or partial response to platinum-based chemotherapy.

- Olaparib (maintenance therapy) was approved August 17, 2017, for maintenance in patients with recurrent ovarian cancer who are in a partial or complete response to platinum-based chemotherapy.

Single-agent studies of PARP inhibitors have focused on patients with BRCA mutations. Response and clinical benefit rates have been high, but in some studies patients without BRCA mutations have also been shown to benefit. A pivotal phase II study provided clinical evidence of olaparib activity in patients with and without BRCA mutations. ${ }^{1}$ However, approval of the drug was for patients with germline BRCA mutations based on a phase II trial in this population showing a response rate of $46 \%$ in patients with platinum-sensitive disease and 30\% in those with platinum-resistant disease. ${ }^{2}$ Median response duration was approximately 8 months in each subset. ${ }^{2}$ The BRCAAnalysis CDx companion diagnostic (Myriad Genetics) was also FDA-approved.

Rucaparib received accelerated approval after the phase II ARIEL2 study showed a response rate of $69 \%$ in patients with germline or somatic BRCA mutations and 30\% in those with "BRCA mutant-like" genomic signatures (loss of heterozygosity presumably due to homologous repair deficiency [HRD]); median PFS was 9.4 and 7.1 months, respectively. These results were compared with a $13 \%$ response rate and 3.7 month PFS in patients who were negative for biomarkers. ${ }^{3} \mathrm{CDx}_{\mathrm{BRCA}}$ assay (Foundation Medicine) is the official FDA-approved companion diagnostic for this, but per the NCCN Guidelines, other assays are also acceptable.

"The [FDA] approval was for patients with either germline or somatic BRCA mutations, and it led to an increase in genomic testing of tumors to potentially identify the group of patients who might benefit," Dr. Armstrong noted.

\section{New: Maintenance PARP Inhibitors}

"The biggest issue in ovarian cancer that's changed in the last couple of years is the use of PARP inhibitors as maintenance therapy," Dr. Armstrong indicated. "Although most of the benefit will be seen in those with BRCA mutations or HRD positivity, even patients without these markers can still benefit."

In a randomized double-blind phase II study in patients with platinum-sensitive high-grade serous ovarian cancer who had received at least 2 previous platinum regimens and were currently experiencing lasting response to platinum-based therapy, maintenance treatment with olaparib (until progression) reduced the risk of progression by $65 \%$ and nearly doubled the overall PFS time. ${ }^{4}$ Results were even more striking in patients with BRCA-mutations, whose median PFS was 11.2 months with maintenance olaparib versus 4.3 months with placebo (HR, 0.18; $P<.00001)$. Interestingly, patients with wild-type mutations also derived a significant benefit (for PFS: HR, 0.53; $P=.007$ ), even though the median PFS times were similar (5.6 and 5.5 months, respectively).

The newer agent niraparib as maintenance yielded benefit in all patients included in the phase III NOVA trial. ${ }^{5}$ Patients in this trial were required to have platinum-sensitive disease, have received at least 2 prior platinum regimens, be in a complete or partial response to the most recent platinum-based regimen, and have serous high-grade histology or germline BRCA mutation. Median PFS was 21.0 versus 5.5 months with niraparib versus placebo (HR, 0.27 ; $<.001$ ) in patients with germline BRCA mutations; 12.9 versus 3.8 months ( $\mathrm{HR}, 0.38 ; P<.001)$ in patients with $H R D, B R C A$ wild-type disease; and 9.3 versus 3.9 months $(\mathrm{HR}, 0.45 ; \mathrm{P}<.001)$ in patients lacking both these markers. These results led to the FDA approval of this drug as maintenance therapy for all patients with platinum-sensitive disease who are in a complete or partial response to platinumbased therapy.

\section{Toxicities of PARP Inhibitors}

"There are toxicities specific to PARP inhibitors," Dr. Armstrong continued. All PARP inhibitors can produce hematologic toxicity, which can be problematic, especially in patients on maintenance therapy who have already been treated with a myelosup- 
pressive regimen. With niraparib, thrombocytopenia can be a particular problem, and grade 3/4 thrombocytopenia was seen in approximately one-third of patients in the NOVA trial. Gastrointestinal toxicities are common, but high grades are not.

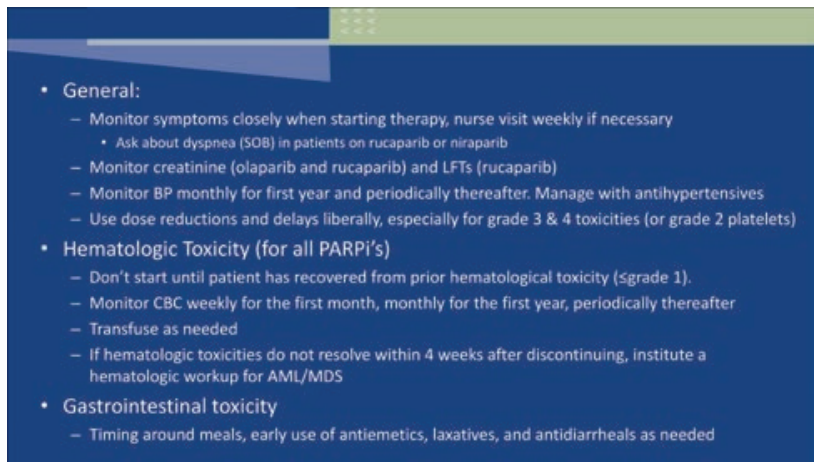

Figure 2. Managing PARPi toxicities: guidelines from Dr. Armstrong's practice.

Abbreviations: AML/MDS, acute myeloid leukemia/myelodysplastic syndromes; BP, blood pressure; LFTs, liver function tests; PARPi, PARP inhibitors; SOB, shortness of breath.
Some toxicities differ among the 3 agents. Increased serum creatinine is seen with some of these agents. The increased creatinine clearance does not, however, represent a change in glomerular filtration rate, she added.

Hepatic toxicity is mostly seen with rucaparib, but are usually transient and respond to dose modification. Hypertension is a little bit more common with niraparib and usually can be managed. Unexplained dyspnea has been seen in at least 2 of these agents.

Dr. Armstrong shared the guidelines she has issued to the nursing staff at her institution (Figure 2). She emphasized the need for close monitoring when therapy is started. At her institution, they use dose reductions or delays liberally, especially for highgrade toxicities and for grade 2 or higher thrombocytopenia. In her practice, they prefer to wait until the patient has recovered from the last cycle of chemotherapy before starting maintenance therapy.

\section{References}

1. Gelmon KA, Tischkowitz M, Mackay H, et al. Olaparib in patients with recurrent high-grade serous or poorly differentiated ovarian carcinoma or triple-negative breast cancer: a phase 2, multicentre, open-label, nonrandomised study. Lancet Oncol 2011;12:852-861.

2. Domchek SM, Aghajanian C, Shapira-Frommer R, et al. Efficacy and safety of olaparib monotherapy in germline BRCA1/2 mutation carriers with advanced ovarian cancer and three or more lines of prior therapy. Gynecol Oncol 2016;140:199-203.
3. Swisher EM, Lin KK, Oza AM, et al. Rucaparib in relapsed, platinumsensitive high-grade ovarian carcinoma (ARIEL2 Part 1): an international, multicentre, open-label, phase 2 trial. Lancet Oncol 2017;18:75-87.

4. Ledermann J, Harter P, Gourley C, et al. Olaparib maintenance therapy in platinum-sensitive relapsed ovarian cancer. N Engl J Med 2012;366:1382_ 1392.

5. Mirza MR, Monk BJ, Herrstedt J, et al. Niraparib maintenance therapy in platinum-sensitive, recurrent ovarian cancer. N Engl J Med 2016;375:2154-2164. 